\title{
Erratum: Theta Functions from a Geometric Viewpoint
}

\section{Jun-Inchi Igusa}

(C) Springer-Verlag Berlin Heidelberg, 1972

J.-i. Igusa, Theta Functions,

DOI 10.1007/978-3-642-65315-5

Erratum to:

Chapter II in: J.-i. Igusa, Theta Functions from a Geometric Viewpoint DOI 10.1007/978-3-642-65315-5_2

The original version of Chapter 2 on page 54 has incorrect content. The chapter has been corrected.

The updated online version of the original chapter can be found at DOI 10.1007/978-3-642-65315-5_2.

J.-i. Igusa, Theta Functions, DOI 10.1007/978-3-642-65315-5_6,

(C) Springer-Verlag Berlin Heidelberg, 2018 